\title{
LA SOLUCIÓN NUTRITIVA MODIFICA EL CRECIMIENTO DE DOS ESPECIES DE ORQUÍDEAS
}

\section{THE NUTRIENT SOLUTION MODIFIES THE GROWTH OF TWO ORCHID SPECIES}

\author{
Nadia Jiménez-Peña ${ }^{1}$, Manuel Sandoval-Villa', Víctor H. Volke-Haller ${ }^{1}$, \\ Martha Pedraza-Santos ${ }^{2}$ y Ma. Teresa Colinas-León ${ }^{3}$
}

\begin{abstract}
'Colegio de Postgraduados, Campus Montecillo, Montecillo, Texcoco, Estado de México, México. ${ }^{2}$ Universidad Michoacana de San Nicolás de Hidalgo, Facultad de Agrobiología, Uruapan, Michoacán, México. 3Universidad Autónoma Chapingo, Departamento de Fitotecnia, Chapingo, Estado de México, México.
\end{abstract}

*Autor para correspondencia (jimenez.nadia@colpos.mx)

\section{RESUMEN}

Las orquídeas (Orchidaceae) se desarrollan en una gran variedad de condiciones ambientales, por lo que tienen requerimientos específicos de nutrición. En la nutrición de orquídeas existe desconocimiento de los efectos de los fertilizantes y de su uso a lo largo del ciclo de estas plantas. El objetivo de esta investigación fue estudiar el efecto de tres soluciones nutritivas sobre el desarrollo y crecimiento de Laelia autumnalis y Paphiopedilum insigne. El experimento se realizó en un periodo de 27 meses en condiciones de invernadero. Las variables evaluadas fueron número de hojas, área foliar, peso fresco y seco total de la planta, de raíz y de hoja, y lecturas SPAD en hojas maduras; además, en $L$. autumnalis se evaluó el número de pseudobulbos, grosor y largo de pseudobulbos, así como el peso fresco y seco de éstos. Se encontró que en $L$. autumnalis la solución de Steiner incrementó el número de hojas (100\%), pseudobulbos (133\%), área foliar ( $80 \%$ ), peso fresco total (78\%) y peso seco total $(125 \%)$, en comparación con las plantas tratadas sólo con agua (testigo). En P. insigne la solución Hoagland-Arnon mejoró el crecimiento vegetativo, incrementó el número de hojas (65\%), área foliar $(83 \%)$, lecturas SPAD (79\%), peso fresco total (33\%) y peso seco total (27 $\%)$, en comparación con las plantas testigo. Los resultados demuestran que las soluciones nutritivas influyen positivamente en el crecimiento de las dos especies de orquídeas y que cada especie tiene requerimientos nutrimentales particulares; asimismo, los resultados podrían ser de utilidad en programas de conservación y aprovechamiento de estas especies.

Palabras clave: Laelia autumnalis, Paphiopedilum insigne, HoaglandArnon, nutrición mineral, Steiner, Wynd.

\section{SUMMARY}

Orchids (Orchidaceae) develop in a wide range of environmental conditions, so they have specific nutrition requirements. In the nutrition of orchids there is lack of knowledge on the effects of fertilizers and their use throughout the cycle of these plants. The objective of this research was to study the effect of three nutrient solutions on the development and growth of Laelia autumnalis and Paphiopedilum insigne. The experiment was conducted over a period of 27 months under greenhouse conditions. The variables evaluated were number of leaves, leaf area, fresh and dry total weight of the plant, root and leaf, and SPAD readings in mature leaves; in addition, in $L$. autumnalis the number of pseudobulbs, thickness and length, as well as the fresh and dry weight of these pseudobulbs were evaluated. It was found that in $L$. autumnalis the Steiner solution increased the number of leaves $(100 \%)$, pseudobulbs (133\%), leaf area (80 \%), total fresh weight (78\%) and total dry weight (125\%), compared to plants treated only with water (control). In $P$. insigne the Hoagland-Arnon solution improved the vegetative growth, increased the number of leaves $(65$ $\%)$, leaf area ( $83 \%)$, SPAD readings ( $79 \%$ ), total fresh weight (33\%) and total dry weight $(27 \%)$, compared to the control plants. Results show that nutrient solutions positively influence growth of the two orchid species, and that each species has particular nutritional requirements; likewise, these results could be useful in conservation and exploitation programs of these species.

Index words: Laelia autumnalis, Paphiopedilum insigne, HoaglandArnon, mineral nutrition, Steiner, Wynd.

\section{INTRODUCCIÓN}

La floricultura desempeña un papel cada vez más importante en la economía mundial actual (Getu, 2009); destacan rosa (Rosa spp.), lili (Lilium spp.), crisantemo (Chrysanthemum spp.), clavel (Dianthus caryophyllus) y las orquídeas (Orchidaceae) como las flores más cultivadas en el mundo (Tse-Leow y Khye-Tan, 2007). Se estima que las orquídeas representan alrededor del $10 \%$ del comercio internacional de flores de corte y maceta (De et al., 2014).

México alberga alrededor de 1260 especies de orquídeas pertenecientes a 170 géneros (Hágsater et al., 2005). L. autumnalis, orquídea nativa de México, destaca por su importancia como planta ornamental y medicinal en varias regiones de nuestro país (Emeterio-Lara et al., 2016). L. autumnalis es una planta epífita que presenta flores púrpuras vistosas de diferentes tonalidades, formas y tamaños (Hernández-Muñoz et al., 2017), con pseudobulbos oblongos, alargados y longitudinalmente arrugados e inflorescencias de hasta $70 \mathrm{~cm}$ de largo, con un racimo de 5 a 12 flores que abren en sucesión; el género consiste de 22 especies, 11 de ellas se encuentran en México (Halbinger y Soto, 1997); sin embargo, las poblaciones naturales han sido fuertemente afectadas debido a la destrucción de su hábitat y extracción excesiva 
(Ávila-Díaz et al., 2009), a pesar de estar protegidas por la Norma Oficial Mexicana NOM-059 (SEMARNAT, 2010). Otro género de orquídeas de importancia comercial y ornamental es el conocido como zapatilla de dama (Paphiopedilum spp.), éste comprende alrededor de 96 especies, principalmente con hábito terrestre, nativas de regiones tropicales y subtropicales del sureste de Asia (Wang et al., 2017a), cuyas flores hermosas y llamativas contribuyen significativamente a la industria de la floricultura; sin embargo, su popularidad como plantas ornamentales ha llevado a la sobre recolección de especies silvestres con fines comerciales, lo que ha provocado que algunas especies se encuentran en peligro de extinción y hayan sido puesta en el CITES (Convención sobre el Comercio Internacional de Especies Amenazadas de Fauna y Flora Silvestres) (Zhang et al., 2016).

Además del comercio ilegal y destrucción de sus hábitats, las orquídeas presentan problemas en su manejo, la nutrición es uno de los más importantes, ya que existe poca información sobre el uso, época, forma y suministro eficiente de los nutrimentos durante su desarrollo, que permita acelerar el crecimiento vegetativo de las plántulas, aumentar la floración temprana y prolongar la vida de florero. Al respecto, Wang y Konow (2002) encontraron que la fertilización de plantas de Phalaenopsis con 200 $\mathrm{mg} \mathrm{L}^{-1}$ de $\mathrm{N}$ y $160 \mathrm{mg} \mathrm{L}^{-1}$ de $\mathrm{K}$ mejora el crecimiento de las plantas desarrolladas en dos sustratos, indican que estas concentraciones son las adecuadas para obtener un excelente crecimiento en dicho género. Wang (2007) indicó que la aplicación de $300 \mathrm{mg} \mathrm{L}^{-1}$ de $\mathrm{K}$ con suministro elevado de $\mathrm{N}$ y P, independientemente del medio, permite obtener plantas de Phalaenopsis de alta calidad, con mayor longitud de hojas y mayor número y tamaño de flores. Por su parte, Tse-Leow y Khye-Tan (2007) demostraron que mediante el uso de un sistema hidropónico automatizado y fertilizando con solución nutritiva (CE de $1 \mathrm{dS}^{-1}$ ), en un híbrido de Dendrobium para flor de corte, se obtienen más pseudobulbos, hojas y flores, además de evitar la pudrición de las raíces, en comparación con los métodos tradicionales de producción. La mayoría de los estudios sobre fertilización en orquídeas se han centrado en híbridos del género Phalaenopsis (Wang y Chang, 2017), Dendrobium (Liu et al., 2014), Cattleya y Cymbidium (Naik et al., 2009), y recientemente, en el género Laelia (JiménezPeña et al., 2013; Rodrigues et al., 2010), mientras que el conocimiento sobre la nutrición de otras especies con potencial ornamental es limitado.

Por lo anteriormente expuesto, la presente investigación tuvo como objetivo evaluar los efectos de tres soluciones nutritivas sobre el desarrollo y crecimiento de dos especies de orquídeas, L. autumnalis y $P$. insigne.

\section{MATERIALES Y MÉTODOS}

\section{Material vegetal}

Se utilizaron plantas de L. autumnalis, las cuales fueron proporcionadas por el banco de germoplasma del Sistema Nacional de Recursos Fitogenéticos (SINAREFI), a partir de plantas que fueron rescatadas de áreas de bosque de encino que habían sido deforestadas en el municipio de Uruapan, Michoacán, mientras que las plantas de $P$. insigne se obtuvieron con un proveedor comercial de la misma ciudad.

\section{Tratamientos}

Se tuvieron ocho tratamientos, los cuales se generaron con la combinación de plantas de dos especies ( $L$. autumnalis y $P$. insigne) irrigadas con tres soluciones y un testigo; 1) solución Hoagland-Arnon (1938), 2) solución Steiner (1984), 3) solución Wynd (1933) tomadas de Hewitt (1966) (Cuadro 1), se utilizaron sales comerciales y 4) plantas irrigadas con agua de pozo (testigo). La concentración de los diferentes elementos en la solución nutritiva se ajustó a partir de los resultados del análisis químico realizado al agua de pozo (Cuadro 2); el pH se ajustó con ácido sulfúrico entre 5.5 y 6.0 .

\section{Manejo del experimento}

El experimento se realizó en condiciones de invernadero, con humedad relativa promedio mensual de $70 \%$ y temperatura promedio $25^{\circ} \mathrm{C}$. Se colocó una malla con sombreo de $60 \%$ para evitar exceso de radiación y se utilizó un sistema de aspersión automatizado que se usaba durante el día para mantener fresco el ambiente. Durante los meses más fríos (noviembre-febrero) se utilizó calefacción por la noche para mantener la temperatura por encima de $11{ }^{\circ} \mathrm{C}$. Las plantas se trasplantaron en macetas oscuras de plástico de $15.24 \mathrm{~cm}$ de diámetro, en $L$. autumnalis cada unidad (una planta) contaba con 4-5 pseudobulbos. El sustrato fue una mezcla de carbón vegetal + tezontle + corteza de pino + turba-vermiculita en una relación de 1:2:2:1, que contenía $64 \%$ de porosidad total, $26 \%$ de retención de agua y 38 \% de capacidad de aireación. Durante los primeros tres meses después del transplante las plantas sólo se irrigaron con agua de pozo, con la finalidad de proporcionar un periodo de adaptación a las condiciones del invernadero. Las plantas se irrigaron cada semana o cuando la superficie del sustrato se encontraba seca, con $130 \mathrm{~mL}$ de solución nutritiva y un riego semanal con agua con la finalidad de evitar la acumulación de sales en el sustrato. 
Cuadro 1. Concentración $\left(\mathrm{mg} \mathrm{L}^{-1}\right)$ de las soluciones nutritivas evaluadas en el crecimiento L. autumnalis y $P$. insigne.

\begin{tabular}{lccc}
\hline Nutrimento & Hoagland-Arnon & Steiner & Wynd \\
\hline $\mathrm{N}$ & 210 & 167 & 225 \\
$\mathrm{P}$ & 31 & 31 & 137 \\
$\mathrm{~K}$ & 235 & 277 & 112 \\
$\mathrm{Ca}$ & 160 & 183 & 169 \\
$\mathrm{Mg}$ & 49 & 49 & 25 \\
$\mathrm{~S}$ & 64 & 67 & 24 \\
$\mathrm{Fe}$ & 5 & 3 & \\
$\mathrm{Mn}$ & & 1.97 & \\
$\mathrm{~B}$ & & 0.44 & \\
$\mathrm{Zn}$ & & 0.11 & \\
$\mathrm{Cu}$ & & 0.02 & \\
$\mathrm{Mo}$ & & 0.007 & \\
\hline
\end{tabular}

Cuadro 2. Análisis químico del agua de pozo $\left(\mathrm{mg} \mathrm{L}^{-1}\right)$ utilizada para preparar las soluciones nutritivas.

\begin{tabular}{lccccccccccc}
\hline Época & $\mathrm{Ca}^{2+}$ & $\mathrm{Mg}^{2+}$ & $\mathrm{K}^{+}$ & $\mathrm{Na}^{+}$ & $\mathrm{H}_{2} \mathrm{PO}_{4}^{-}$ & $\mathrm{SO}_{4}^{2+}$ & $\mathrm{MoO}_{4}^{2-}$ & $\mathrm{Fe}^{2+}$ & $\mathrm{Mn}^{2+}$ & $\mathrm{Zn}^{2+}$ & $\mathrm{H}_{3} \mathrm{BO}_{3}$ \\
\hline Lluvia & 22.5 & 21.4 & 1.2 & 37.4 & 0.17 & 16.8 & - & - & - & - & - \\
Seca & 27.2 & 30.7 & 3.9 & 39.4 & 0.20 & 18.5 & 0.03 & 0.13 & 0.02 & 0.06 & 0.06 \\
\hline
\end{tabular}

Variables de estudio

En ambas especies, durante el primer año se evaluó el número de hojas, mientras que en el segundo año se evaluó número de hojas, área foliar, peso fresco y seco total de la planta, raíz y hoja, y lecturas SPAD en hojas maduras por unidad experimental (maceta). Adicionalmente, dado que L. autumnalis presenta estructuras llamadas pseudobulbos como tallos, también se evaluó el número, grosor y largo de pseudobulbos. El peso fresco de raíces, hojas y pseudobulbos por maceta, al final del periodo de evaluación y se secaron en una estufa con aire circulante a $70{ }^{\circ} \mathrm{C}$ marca Riossa (Modelo HFC-125D, Monterrey, N. L., Mexico). Para determinar el área foliar se utilizó un medidor de área foliar marca LI-COR (Modelo LI-3100, Lincoln, Nebraska, USA). Las lecturas SPAD se realizaron con un SPAD-502 (Konica Minolta, Osaka, Japón).

\section{Diseño experimental y análisis de datos}

Se empleó un diseño experimental completamente al azar y la unidad experimental consistió en una planta por maceta. $L$. autumnalis tuvo 12 repeticiones y $P$. insigne 16 repeticiones por tipos de nutrición (tres soluciones nutritivas más el testigo). Los datos obtenidos se analizaron mediante análisis de varianza y comparación de medias de Tukey $(P \leq 0.05)$ con el programa estadístico SAS para Windows v9.1 (SAS Institute, 2003).

\section{RESULTADOS}

\section{Desarrollo vegetativo}

Para el primer año de evaluación L. autumnalis presentó diferencias significativas con la aplicación de las tres soluciones nutritivas evaluadas, en comparación a las plantas testigo (Cuadro 3). El número de hojas se incrementó con las soluciones Wynd, Hoagland-Arnon y Steiner en 26, 18 y $30 \%$, respectivamente. El ancho y largo de pseudobulbos se incrementó al usar las soluciones nutritivas Wynd y Hoagland-Arnon. En el segundo año de evaluación las tres soluciones incrementaron significativamente $(P \leq 0.05)$ el desarrollo de $L$. autumnalis; el mayor desarrollo de hojas (114\%) se observó con la solución Wynd, mientras que el número de pseudobulbos ocurrió con la solución Steiner (133 \%) en comparación con las plantas testigo. El área foliar se afectó en $L$. autumnalis como repuesta a la aplicación de las soluciones nutritivas, donde la solución Wynd fue la que generó el mayor incremento en esta variable (123\%) (Cuadro 3).

La aplicación de las soluciones nutritivas Wynd, Hoagland-Arnon y Steiner incrementó el número de hojas en plantas de P. insigne; la solución de Hoagland-Arnon generó incrementos en ambos años del estudio de 90 y 65 $\%$ en comparación con las plantas testigo. El área foliar se incrementó 105 \% en P. insigne con la solución Steiner con 
respecto a las plantas testigo (Cuadro 4).

\section{Lecturas SPAD}

En $L$. autumnalis las lecturas SPAD fueron inferiores en las plantas fertilizadas, en comparación a las irrigadas con agua, las cuales mostraron los mayores valores (Cuadro 3); sin embargo, en P. insigne las lecturas SPAD se incrementaron con la aplicación de las soluciones nutritivas, la solución de Hoagland-Arnon produjo un valor significativamente mayor (34.16) en comparación con el testigo (19.02) (Cuadro 4).

\section{Peso fresco y seco}

En L. autumnalis las variables de peso fresco y seco total se incrementaron en aquellas plantas irrigadas con las soluciones Wynd y Steiner, mientras que las irrigadas con la solución Hoagland-Arnon mostraron valores bajos y similares a los de las plantas testigo. El peso fresco de raíz, de pseudobulbos, de hojas y el peso fresco total en plantas de L. autumnalis irrigadas con la solución Steiner se incrementó en 34, 76, 202 y $78 \%$, respectivamente, mientras que las irrigadas con la solución Wynd tuvieron incremento de 16, 13, 130 y $20 \%$ respectivamente en comparación con las irrigadas con agua. El peso seco total también se incrementó significativamente con la aplicación de la solución Wynd y Steiner con incremento de 135 y $147 \%$ en comparación con las plantas testigo, aunque las tratadas con la solución Hoagland-Arnon tuvieron un comportamiento similar o inferior al de las plantas irrigadas sólo con agua (Cuadro 5).

Las plantas de $P$. insigne irrigadas con las soluciones nutritivas Hoagland-Arnon y Steiner mostraron los mayores incrementos para peso fresco total con 34 y $40 \%$,

Cuadro 3. Efecto de soluciones nutritivas en el crecimiento y lecturas SPAD de la orquídea $L$. autumnalis evaluada por dos años.

\begin{tabular}{lccccc}
\hline \multirow{2}{*}{ Variable } & \multirow{2}{*}{ Valor de P } & \multicolumn{4}{c}{ Solución nutritiva } \\
\cline { 3 - 5 } & & Testigo & Wynd & Hoagland-Arnon & Steiner \\
\hline & & & Desarrollo vegetativo primer año \\
Número de hojas & 0.0255 & $2.17 \mathrm{~b}$ & $2.75 \mathrm{ab}$ & $2.58 \mathrm{ab}$ & $2.83 \mathrm{a}$ \\
Número de pseudobulbos & 0.0001 & $2.00 \mathrm{a}$ & $1.00 \mathrm{~b}$ & $1.00 \mathrm{~b}$ & $1.00 \mathrm{~b}$ \\
Ancho pseudobulbos (mm) & 0.0001 & $18.72 \mathrm{~b}$ & $26.15 \mathrm{a}$ & $24.82 \mathrm{a}$ & $21.28 \mathrm{~b}$ \\
Largo pseudobulbos (mm) & 0.0006 & $7.52 \mathrm{~b}$ & $9.36 \mathrm{a}$ & $9.73 \mathrm{a}$ & $10.19 \mathrm{a}$ \\
& & & Desarrollo vegetativo segundo año & \\
Número de hojas & 0.0013 & $3.00 \mathrm{~b}$ & $6.43 \mathrm{a}$ & $5.67 \mathrm{a}$ & $6.00 \mathrm{a}$ \\
Número de pseudobulbos & 0.0134 & $1.50 \mathrm{~b}$ & $3.14 \mathrm{a}$ & $3.40 \mathrm{a}$ & $3.50 \mathrm{a}$ \\
Ancho pseudobulbos (mm) & $\leq 0.0500$ & $21.00 \mathrm{ab}$ & $17.99 \mathrm{~b}$ & $15.59 \mathrm{~b}$ & $19.36 \mathrm{a}$ \\
Largo psuedobulbos (mm) & 0.0001 & $91.97 \mathrm{a}$ & $70.52 \mathrm{bc}$ & $49.93 \mathrm{bcd}$ & $81.68 \mathrm{~b}$ \\
Área foliar (cm ${ }^{2}$ ) & $\leq 0.0500$ & $122.36 \mathrm{~b}$ & $273.57 \mathrm{a}$ & $170.81 \mathrm{ab}$ & $221.47 \mathrm{ab}$ \\
Lecturas SPAD & 0.0019 & $75.70 \mathrm{a}$ & $63.68 \mathrm{~b}$ & $58.28 \mathrm{bc}$ & $69.37 \mathrm{ab}$ \\
\hline
\end{tabular}

Medias con letras iguales en las hileras no son estadísticamente diferentes (Tukey, 0.05).

Cuadro 4. Efecto de soluciones nutritivas en el crecimiento y lectura SPAD de la orquídea $P$. insigne evaluada por dos años.

\begin{tabular}{lccccc}
\hline \multirow{2}{*}{ Variable } & Valor de P & \multicolumn{4}{c}{ Solución nutritiva } \\
\cline { 3 - 6 } & & Testigo & Wynd & Hoagland-Arnon & Steiner \\
\hline & & & Desarrollo vegetativo \\
Número de hojas primer año & 0.0138 & $3.25 \mathrm{~b}$ & $6.67 \mathrm{a}$ & $6.20 \mathrm{a}$ & $5.69 \mathrm{ab}$ \\
Número de hojas segundo año & 0.0164 & $8.55 \mathrm{~b}$ & $8.31 \mathrm{~b}$ & $14.13 \mathrm{a}$ & $10.11 \mathrm{ab}$ \\
Área foliar $\left(\mathrm{cm}^{3}\right)$ & 0.0474 & $263.84 \mathrm{~b}$ & $477.42 \mathrm{ab}$ & $483.38 \mathrm{ab}$ & $541.11 \mathrm{a}$ \\
Lecturas SPAD & 0.0001 & $19.02 \mathrm{bc}$ & $29.23 \mathrm{~b}$ & $34.16 \mathrm{a}$ & $31.69 \mathrm{ab}$ \\
\hline
\end{tabular}

Medias con letras iguales en las hileras no son estadísticamente diferentes (Tukey, 0.05) 
respectivamente, en comparación con las plantas testigo; la solución Steiner generó incremento en peso seco total de $24 \%$ en comparación con las irrigadas con agua; sin embargo, en raíces se observó que el peso fresco y seco disminuyó con la aplicación de soluciones nutritivas (Cuadro 6). En general, se observaron plantas vigorosas, verdes y con más hojas en los tratamientos con soluciones nutritivas, mientras que en las irrigadas sólo con agua las plantas mostraron clorosis y menor tamaño (Figura 2).

\section{DISCUSIÓN}

Las soluciones Wynd, Hoagland-Arnon y Steiner incrementaron el crecimiento de la orquídea epífita $L$. autumnalis y de la terrestre $P$. insigne, en comparación con las plantas testigo (Figuras 1 y 2), lo que generó plantas con mayor calidad. Lo anterior puede deberse a que el agua sólo aportó a las plantas los nutrimentos que contenía en estado natural; es decir, pequeñas cantidades de algunos macro y micronutrimentos (Cuadro 2), mientras que las soluciones nutritivas aportaron elementos esenciales en concentraciones y proporciones adecuadas para lograr un mejor crecimiento de las plantas. Lo anterior puede deberse a las elevadas concentraciones de $\mathrm{N}$ y K presentes en las soluciones nutritivas. Se ha observado que altas concentraciones de $\mathrm{N}$ y $\mathrm{K}$ son más determinantes en la promoción de crecimiento que el $\mathrm{P}$ (Wang, 2007). Wang y Konow (2002) señalaron que la aplicación de fertilizantes con cantidades altas y similares en $\mathrm{N}$ y $\mathrm{K}$, y bajas en P (20N-2.2P-15.8K y 20N-8.6P-16.6K) en orquídeas Phalaenopsis cultivadas en mezcla de corteza-turba incrementa el número de hojas, área foliar y peso fresco

Cuadro 5. Efecto de soluciones nutritivas en peso fresco y seco en estructuras de la orquídea $L$. autumnalis evaluada por dos años.

\begin{tabular}{|c|c|c|c|c|c|}
\hline \multirow{2}{*}{ Estructura } & \multirow{2}{*}{ Valor de P } & \multicolumn{4}{|c|}{ Solución nutritiva } \\
\hline & & Testigo & Wynd & Hoagland-Arnon & Steiner \\
\hline & & \multicolumn{4}{|c|}{ Peso fresco (g) } \\
\hline Hojas & 0.0028 & $9.07 \mathrm{~b}$ & $20.87 a b$ & $11.23 b$ & $27.47 \mathrm{a}$ \\
\hline Pseudobulbos & 0.0301 & $126.97 \mathrm{ab}$ & $143.73 a b$ & $69.57 b$ & $224.23 a$ \\
\hline Raíz & 0.0500 & $19.60 \mathrm{a}$ & 22.73 a & $11.27 \mathrm{a}$ & $26.30 \mathrm{a}$ \\
\hline \multirow[t]{2}{*}{ Total } & 0.0125 & $155.60 \mathrm{~b}$ & 183.6 ab & $92.00 \mathrm{~b}$ & $278.00 \mathrm{a}$ \\
\hline & & \multicolumn{4}{|c|}{ Peso seco (g) } \\
\hline Hojas & 0.0124 & $1.48 b$ & $4.03 \mathrm{ab}$ & $1.57 \mathrm{~b}$ & $5.07 \mathrm{a}$ \\
\hline Pseudobulbos & 0.0233 & $10.07 a b$ & $26.40 \mathrm{a}$ & $6.33 b$ & $25.30 \mathrm{ab}$ \\
\hline Raíz & 0.0500 & $2.40 \mathrm{a}$ & $2.30 \mathrm{a}$ & $2.63 \mathrm{a}$ & $4.10 \mathrm{a}$ \\
\hline Total & 0.0125 & $13.95 b$ & $32.73 a$ & $10.53 b$ & $31.47 \mathrm{a}$ \\
\hline
\end{tabular}

Medias con letras iguales en las hileras no son estadísticamente diferentes (Tukey, 0.05).

Cuadro 6. Efecto de soluciones nutritivas en peso fresco y seco en estructuras de la orquídea $P$. insigne evaluada por dos años.

\begin{tabular}{|c|c|c|c|c|c|}
\hline \multirow{2}{*}{ Estructura } & \multirow{2}{*}{ Valor de P } & \multicolumn{4}{|c|}{ Solución nutritiva } \\
\hline & & Testigo & Wynd & Hoagland-Arnon & Steiner \\
\hline & & \multicolumn{4}{|c|}{ Peso fresco (g) } \\
\hline Hoja & 0.0139 & $22.53 b$ & $27.90 \mathrm{~b}$ & $50.03 a$ & $43.50 \mathrm{a}$ \\
\hline Raíz & 0.0001 & $21.53 \mathrm{a}$ & $5.97 b$ & $8.65 b$ & $11.33 b$ \\
\hline \multirow[t]{2}{*}{ Total } & 0.0272 & $44.06 \mathrm{ab}$ & $33.87 b$ & 58.9 a & $54.83 a$ \\
\hline & & \multicolumn{4}{|c|}{ Peso seco (g) } \\
\hline Hoja & 0.0142 & $3.33 b$ & $5.35 \mathrm{ab}$ & $5.33 a b$ & $6.87 a$ \\
\hline Raíz & 0.0297 & $2.50 \mathrm{a}$ & $1.20 b$ & $2.10 a b$ & $1.77 a b$ \\
\hline Total & 0.0546 & $5.83 a b$ & $6.55 \mathrm{ab}$ & $7.43 a b$ & $8.63 a$ \\
\hline
\end{tabular}

Medias con letras iguales en las hileras no son estadísticamente diferentes (Tukey, 0.05) 


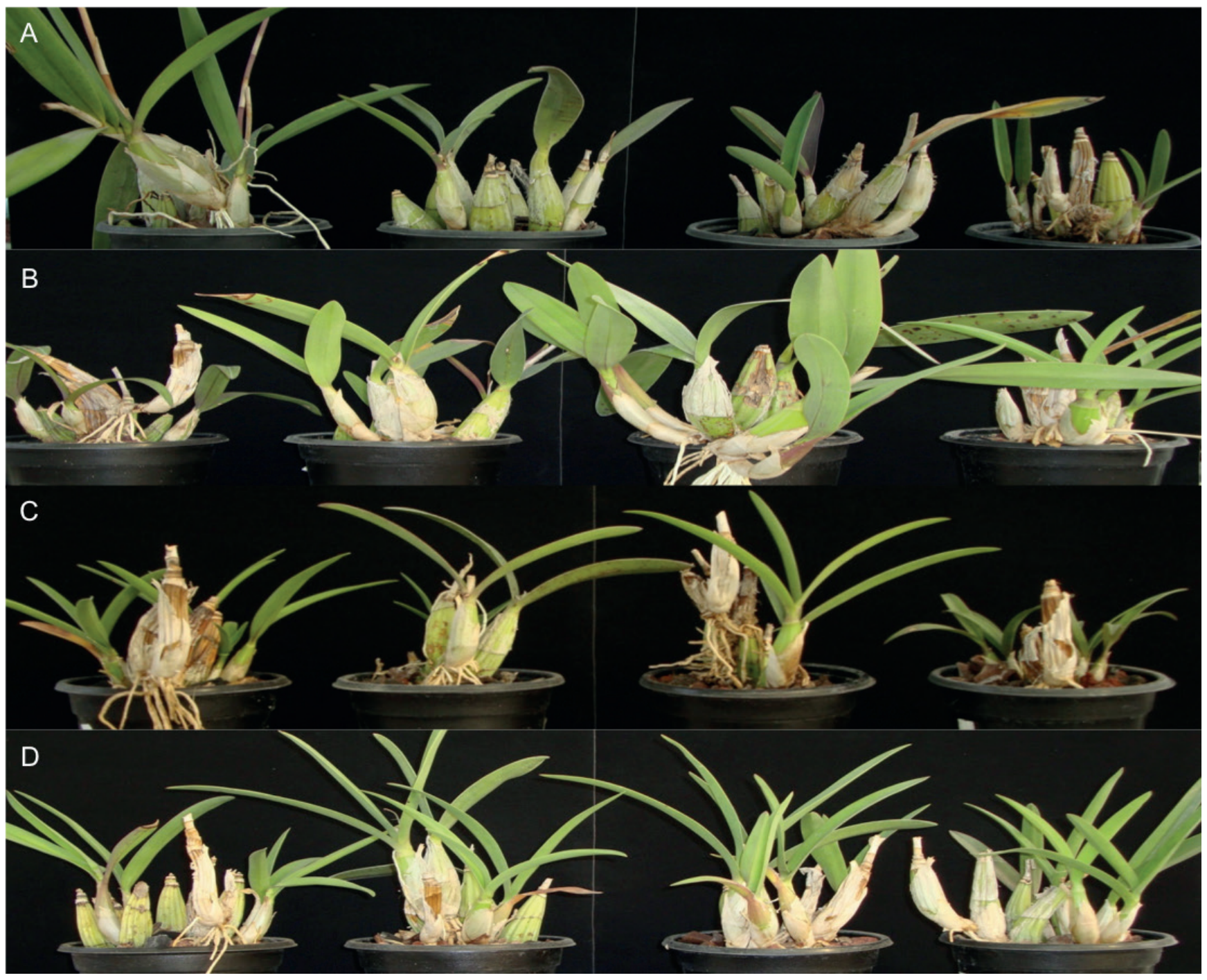

Figura 1. Efecto de soluciones nutritivas en el desarrollo de la orquídea Laelia autumnalis durante un periodo de dos años en condiciones de invernadero. A) agua de pozo, B) solución de Wynd, C) solución de Hoagland-Arnon, D) solución de Steiner.

de follaje y raíz con respecto a los fertilizantes con menor concentración de N y K (2N-0.4P-1.7K).

En otro estudio en Phalaenopsis, el uso de $200 \mathrm{mg} \mathrm{L}^{-1}$ de $\mathrm{N}$, de 25 a $50 \mathrm{mg} \mathrm{L}^{-1}$ de $\mathrm{P}$ y $200 \mathrm{mg} \mathrm{L}^{-1}$ de $\mathrm{K}$ fue suficiente para obtener follaje sano y aumentar el número y tamaño de flores (Wang, 2010), mientras que en Dendrobium el uso de $100 \mathrm{mg} \mathrm{L}^{-1}$ de $\mathrm{N}, 25 \mathrm{mg} \mathrm{L}^{-1}$ de $P$ y $100 \mathrm{mg} \mathrm{L}^{-1}$ de $\mathrm{K}$ se recomienda para obtener un óptimo crecimiento vegetativo y reproductivo (Bichsel et al., 2008). De acuerdo con las concentraciones anteriores, en el presente estudio la solución Hoagland-Arnon tenía concentraciones similares de $\mathrm{N}\left(210 \mathrm{mg} \mathrm{L}^{-1}\right), \mathrm{P}\left(31 \mathrm{mg} \mathrm{L}^{-1}\right)$ y K (235 $\left.\mathrm{mg} \mathrm{L}^{-1}\right)$, mientras que la solución Steiner, además de tener concentraciones altas de $\mathrm{N}$ y $\mathrm{K}$, también contenía microelementos ( $\mathrm{Fe}, \mathrm{Mn}$, $\mathrm{B}, \mathrm{Zn}, \mathrm{Cu}$ y Mo), lo que podría explicar el efecto positivo que tuvieron estas soluciones en el crecimiento de las orquídeas estudiadas.

Durante el primer año del estudio se observó que el largo y grosor de pseudobulbos de L. autumnalis se incrementó con el uso de soluciones nutritivas; sin embargo, en el segundo año las plantas testigo tuvieron los mayores incrementos para estas variables. Al respecto, Bichsel et al. (2008) indicaron que en híbridos de Dendrobium los pseudobulbos son más delgados cuando el tiempo de fertilización o las dosis de N, P y K son mayores, lo que sugiere que la aplicación prolongada de fertilizantes provoca un estado vegetativo prolongado y por lo tanto una maduración tardía del pseudobulbo. Por lo anterior, es probable que la aplicación prolongada de fertilizantes (dos años) en L. autumnalis haya provocado un crecimiento 


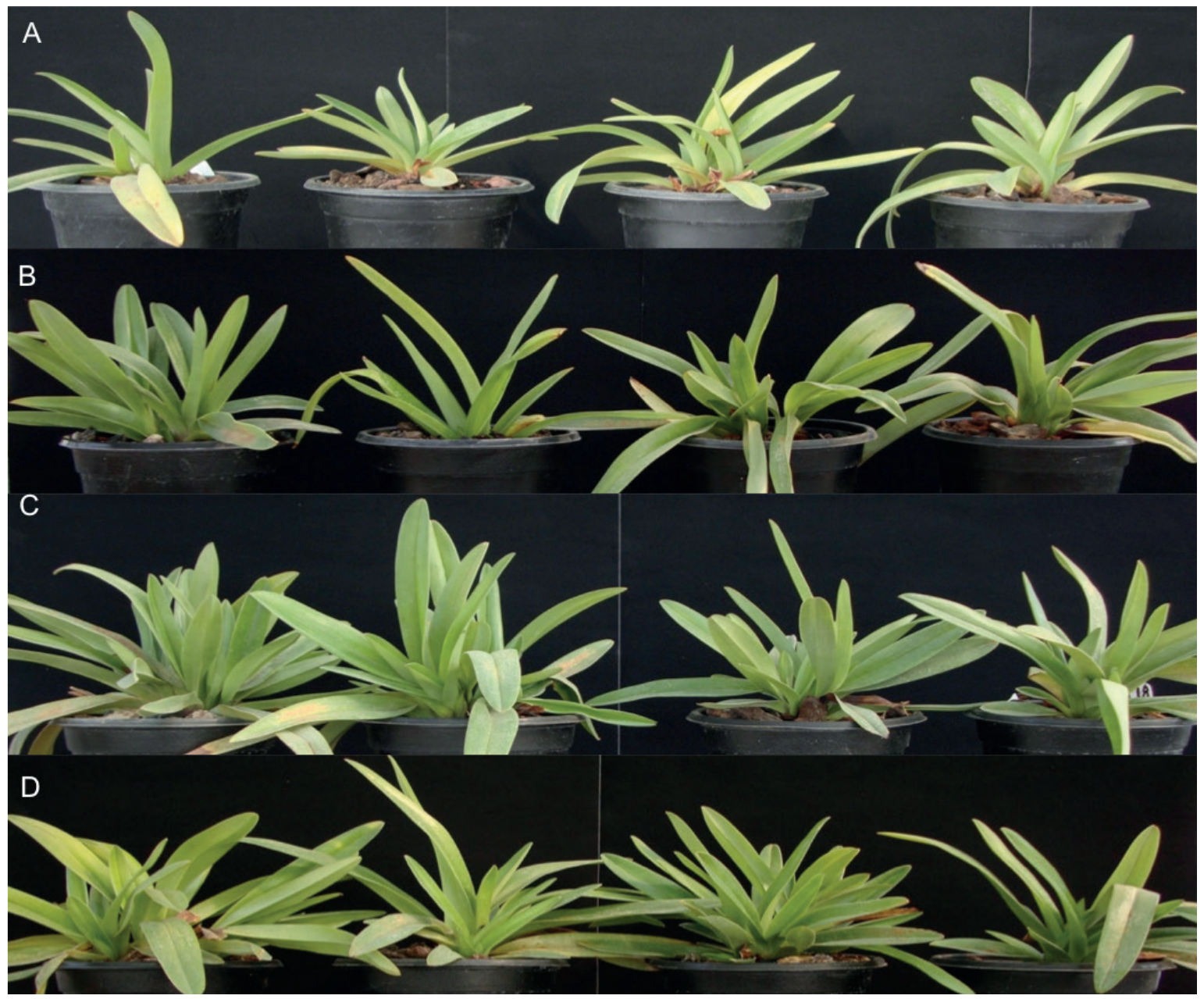

Figura 2. Efecto de soluciones nutritivas en el desarrollo de la orquídea Paphiopedilum insigne durante un periodo de dos años bajo condiciones de invernadero. A) agua de pozo, B) solución de Wynd, C) solución de Hoagland-Arnon, D) solución de Steiner.

vegetativo prolongado, aunado a un mayor número de pseudobulbos, lo cual originó que los pseudobulbos maduraran tardíamente.

En $P$. insigne hubo diferencias en lecturas SPAD, éstas fueron mayores en las plantas irrigadas con las soluciones nutritivas, aunque sólo la solución Hoagland-Arnon mostró diferencias significativas con respecto a las plantas testigo. El índice SPAD es un indicador de concentración de clorofila foliar, y dado que el $\mathrm{N}$ es esencial en la formación de clorofila, éste puede correlacionarse con la concentración de $\mathrm{N}$ en la planta (da Cunha et al., 2015). Por lo anterior, se puede afirmar que el uso de soluciones nutritivas incrementó la concentración N en P. insigne, lo que mejoró su crecimiento. Al respecto, Silva et al. (2013) encontraron un incremento en la concentración de clorofila total en la orquídea L. purpurata, en plantas fertilizadas con $\mathrm{N}$ en forma de urea en condiciones in vitro. Las lecturas SPAD observadas en $P$. insigne (de 19.02 a 34.16) son similares a las reportadas por Liu (2006), quien al evaluar el efecto de diferentes sustratos sobre la tasa fotosintética en $P$. callosum encontró lecturas SPAD que oscilaban de 25.97 a 34.37. Por otra parte, en L. autumnalis las lecturas SPAD fueron mayores en las plantas testigo en comparación con las irrigadas con solución nutritiva, ésto pudo deberse a una posible acumulación de sales en el sustrato, lo que provocó una reducción en la concentración de clorofila.

El peso fresco y seco del follaje en P. insigne se incrementó significativamente con la solución Steiner, mientras que en raíz éste disminuyó significativamente en comparación con las plantas testigo. En L. autumnalis la solución Steiner también incrementó significativamente el peso fresco y seco del follaje y total de la planta; en pseudobulbos la solución Wynd mostró incrementos con respecto al testigo mientras que en la raíz no se observaron diferencias 
significativas en el peso fresco y seco entre los diferentes tratamientos. La menor producción de raíces en plantas de $P$. insigne irrigadas con algunas de las soluciones nutritivas puede deberse a la fertilización constante de las plantas con N, P y K durante un periodo prolongado (dos años), que favoreció la acumulación de sales en el sustrato y redujo el crecimiento de raíces; al respecto Mantovani et al. (2018) mencionaron que plantas de Phalaenopsis y Dendrobium expuestas a estrés por sales (proporciones de $\mathrm{NH}_{4}-\mathrm{N}$ superiores al $75 \%$ ) por periodos prolongados de tiempo (12 meses) reduce el crecimiento de raíces y de la planta; de igual forma, Wang (1998) observó que al irrigar plantas de Phalaenopsis con agua con niveles crecientes de salinidad (CE 1.10 y $1.40 \mathrm{dS} \mathrm{m}^{-1}$ ) el peso fresco de raíces disminuyó con el incremento de la salinidad y favoreció el peso fresco del follaje, tamaño de hojas y número de flores; sin embargo, la respuesta diferencial observada entre $L$. autumnalis y $P$. insigne con respecto al crecimiento de raíz podría sugerir que ambas especies responden de diferente manera a la acumulación de sales por fertilización. Al respecto Franco et al. (2011) señalan que el grado de repuesta al estrés por sales puede variar considerablemente a nivel de familia, género o especie de plantas, lo que afecta el desarrollo de las raíces.

\section{CONCLUSIONES}

El uso de la solución Steiner incrementó en L. autumnalis el número de hojas, pseudobulbos, área foliar, peso fresco y seco total de las plantas. En P. insigne la solución Hoagland-Arnon mejoró el desarrollo vegetativo en el número de hojas, área foliar, peso fresco y peso seco total, lo cual favorece la calidad de estas plantas en maceta. Las soluciones nutritivas influyen positivamente en el desarrollo de estas dos especies de orquídeas y que cada especie tiene requerimientos nutrimentales específicos. Estos resultados pueden servir como base para estructurar programas de conservación y aprovechamiento de las dos especies de orquídeas.

\section{BIBLIOGRAFÍA}

Ávila-Díaz I., K. Oyama, C. Gómez-Alonso and R. Salgado-Garciglia (2009) In vitro propagation of the endangered orchid Laelia speciosa. Plant Cell, Tissue and Organ Culture 99:335-343, https://doi.org/10.1007/s11240-009-9609-8

Bichsel R. G., T. W. Starman and Y. T. Wang (2008) Nitrogen, phosphorus and potassium requirements for optimizing growth and flowering of the nobile Dendrobium as a potted orchid. HortScience 43:328332, https://doi.org/10.21273/HORTSCI.43.2.328

da Cunha A. R., I. Katz, A. P. Souza y R. A. M. Uribe (2015) Índice SPAD en el crecimiento y desarrollo de plantas de lisianthus en función de diferentes dosis de nitrógeno en ambiente protegido. IDESIA (Chile) 33:97-105, https://doi.org/10.4067/S0718-34292015000200012

De L. C., S. P. Vij and R. P. Medhi (2014) Post-harvest physiology and technology in orchids. Journal of Horticulture 1:1-9, https://doi.org/10.4172/2376-0354.1000102
Emeterio-Lara A., V. Palma-Linares, L. M. Vázquez-García y J. Mejía-Carranza (2016) Usos y comercialización de orquídeas silvestres en la región sur del Estado de México. Polibotánica 42:197-214 https://doi.org/10.18387/polibotanica.42.10

Franco J. A., S. Bañón, M. J. Vicente, J. Miralles and J. J. MartínezSánchez (2011) Root development in horticultural plants grown under abiotic stress conditions - a review. Journal of Horticultural Science and Biotecnology 86:543-556, https://doi.org/10.1080/14620316.2011.11512802

Getu M. (2009) Ethiopian floriculture and its impact on the environment: regulation, supervision and compliance. Mizan Law Review 3:240-270, https://doi.org/10.4314/mlr.v3i2.54011

Hágsater E., M. A. Soto A., G. A. Salazar C., R. Jiménez M., M. A. López R. y R. L. Dressler (2005) Las Orquídeas de México. Instituto Chinoín. México, D.F. 304 p, https://doi.org/10.21829/abm75.2006.1132

Halbinger F. y M. Soto (1997) Laelias of Mexico. Orquídea (Méx.) 15:1160.

Hernández-Muñoz S., M. E. Pedraza-Santos, P. A. López, E. De La Cruz-Torres, S. P. Fernández-Pavía, A. Martínez-Palacios y M. Martínez-Trujillo (2017) Determinación de la $\mathrm{DL}_{50}$ y $\mathrm{GR}_{50}$ con rayos gamma $\left({ }^{60} \mathrm{Co}\right)$ en protocormos de Laelia autumnalis in vitro. Agrociencia 51:507-524.

Hewitt E. J. (1966) Sand and Water Culture Methods Used in the Study of Plant Nutrition. 2nd edition. Commonwealth Agricultral Bureaux International. Wallingford, England. 547 p.

Hoagland D. R. and D. I. Arnon (1938) The Water-Culture Method for Growing Plants Without Soil. Circular 347. University of California. Berkeley, California. 39 p.

Jiménez-Peña N., L. A. Valdez-Aguilar, A. M. Castillo-Gónzalez, M. T. ColinasLeón, A. D. Cartmill and D. L. Cartmill (2013) Growing media and nutrient solution concentration affect vegetative growth and nutrition of Laelia anceps Lindl. HortScience 48:773-779, https://doi.org/10.21273/HORTSCI.48.6.773

Liu X. Y. (2006) Effects of growing media on growth and leaf net photosynthetic rate of Paphiopedilum callosum. Southwest China Journal of Agricultural Sciences 19:44-49.

Liu H., Y. B. Luo, J. Heinen, M. Bhat and Z. J. Liu (2014) Eat your orchid and have it too: a potentially new conservation formula for Chinese epiphytic medicinal orchids. Biodiversity and Conservation 23:1215-1228, https://doi.org/10.1007/s10531-014-0661-2

Mantovani C., R. M. Prado and K. F. L. Pivetta (2018) Impact of nitrate and ammonium ratio on nutrition and growth of two epiphytic orchids. Anais da Academia Brasileira de Ciências 90:34233431, https://doi.org/10.1590/0001-3765201820171008

Naik S. K., T. U. Bharathi, D. Barman, R. Devadas, Rampal and R. P. Medhi (2009) Status of mineral nutrition of orchid - a review. Journal of Ornamental Horticulture 12:1-14.

Rodrigues D. T., R. F. Novais, H. Alvarez V., J. M. M. Dias and E. M. A. Villani (2010) Orchid growth and nutrition in response to mineral and organic fertilizers. Revista Brasileira de Ciência do Solo 34:16091616, https://doi.org/10.1590/S0100-06832010000500014

SEMARNAT, Secretaría de Medio Ambiente y Recursos Naturales (2010) Norma Oficial Mexicana NOM-059. Protección ambientalespecies nativas de México de flora y fauna silvestres. Categorías de riesgo y especificaciones para su inclusión, exclusión o cambio. Lista de especies en riesgo. Diario Oficial de la Federación. 30 de septiembre de 2010, Segunda Sección. México, D.F. pp:1-78,

SAS Institute (2003) SAS/STAT User's Guide. Release 9.1. SAS Institute Inc. Cary, North Carolina, USA. $5121 \mathrm{p}$.

Silva J. J. M., M. Rodrigues, E. M. de Castro, S. K. V. Bertolucci and M. Pasqual (2013) Changes in anatomy and chlorophyll synthesis in orchids propagated in vitro in the presence of urea. Acta Scientiarum. Agronomy 35:65-72, https://doi.org/10.4025/actasciagron.v35i1.15356

Steiner A. A. (1984) The universal nutrient solution. In: Proceedings 6th International Congress Soilless Culture. Wageningen, The Netherlands. pp:633-649.

Tse-Leow A. C. and T. K. Khye-Tan (2007) Versatile hydroponic technology for commercial orchid cultivation. Acta Horticulturae 742:75-83, https://doi.org/10.17660/ActaHortic.2007.742.10

Wang Y. T. (1998) Impact of salinity and media on growth and flowering of a hybrid Phalaenopsis orchid. HortScience 33:247-250. 
Wang Y. T. (2007) Potassium nutrition affects Phalaenopsis growth and flowering. HortScience 42:1563-1567, https://doi.org/10.21273/HORTSCI.42.7.1563

Wang Y. T. (2010) Phalaenopsis mineral nutrition. Acta Horticulturae 878:321-333, https://doi.org/10.17660/ActaHortic.2010.878.41

Wang Y. T. and E. A. Konow (2002) Fertilizer source and medium composition affect vegetative growth and mineral nutrition of a hybrid moth orchid. Journal of the American Society for Horticultural Science 127:442-447, https://doi.org/10.21273/JASHS.127.3.442

Wang M., S. R. Lan and Z. J. Liu (2017a) Paphiopedilium notatisepalum, a new species of slipper orchid (Cypripedioideae, Orchidaceae) from China based on morphological and DNA evidence. Phytotaxa
302:156-164, https://doi.org/10.11646/phytotaxa.302.2.5

Wang Y. T. and Y.C. A. Chang (2017) Effects of nitrogen and the various forms of nitrogen on Phalaenopsis orchid - a review. HortTecnology 27:144-149, https://doi.org/10.21273/HORTTECH03204-16

Wynd F. L. (1933) Sources of carbohydrate for germination and growth of orchid seedlings. Annals of the Missouri Botanical Garden 20:569-581, https://doi.org/10.2307/2394196

Zhang F. P., J. L. Huang and S. B. Zhang (2016) Trait evolution in the slipper orchid Paphiopedilium (Orchidaceae) in China. Plant Signaling and Behavior 11:e1149668, https://doi.org/10.1080/15592324.2016.1149668 
